\title{
Serovar distribution of urogenital Chlamydia trachomatis strains in The Netherlands
}

\author{
J H T WAGENVOORT, ${ }^{\ddagger}$ R J SUCHLAND, $\dagger$ W E STAMM $\ddagger$ \\ From the *Department of Clinical Microbiology, University Hospital Rotterdam, The Netherlands, the \\ $\dagger$ Department of Medicine, School of Medicine, and the $\ddagger$ Department of Epidemiology, School of Public Health, \\ University of Washington, Seattle, Washington, USA
}

SUMMARY The distribution of serovars in 208 Chlamydia trachomatis strains of urogenital origin isolated from 185 patients attending a sexually transmitted disease clinic in Rotterdam, The Netherlands, was assessed. Typing by monoclonal antisera using a dot enzyme linked immunosorbent assay (ELISA) showed that the most common serovars were $E$ (found in 45 strains), F (39), D (34), and $\mathrm{K}$ (28). Other serovars detected were $\mathrm{H}(21), \mathrm{G}, \mathrm{I}, \mathrm{I}^{\prime}, \mathrm{J}$ (two to 12), and B (one strain). Mixed infection with two serovars was detected in two patients. These results indicate that most genital infections with $C$ trachomatis result from a small number of serovars, and that those serovars are similar in The Netherlands and Seattle, USA.

Chlamydia trachomatis has been recognised as an important sexually transmitted pathogen with worldwide distribution..$^{1-4}$ Using a microimmunofluorescence method, Wang et al originally defined 15 different Chlamydia trachomatis serovars.' Serovar identification has subsequently served as the basis for studies associating specific $C$ trachomatis serovars with particular sites of infection. The technical difficulty and expense of this typing method, however, has prevented its widespread application in large epidemiological studies. More recently, Wang et al have developed a panel of monoclonal antibodies that greatly simplifies $C$ trachomatis serotyping. ${ }^{6}$ Barnes $e t$ al have adapted these monoclonal antibodies to a solid phase enzyme linked immunosorbent assay (ELISA) of the dot type, ${ }^{7}$ a technique that is reliable, rapid, simple, easy to interpret, and well suited to simultaneously serotyping large numbers of isolates.

Using this method, we assessed the distribution of $C$ trachomatis serovars in 208 cervical and urethral isolates from 185 patients attending a large outpatient clinic for sexually transmitted diseases in Rotterdam, The Netherlands. These findings can serve as a basis for comparison with serotyping results obtained elsewhere and may be useful in elucidating geographical or other epidemiological patterns.

Address for reprints: Dr J H T Wagenvoort, Department of Clinical Microbiology, University Hospital Rotterdam, Dijkzigt 3015 GD Rotterdam, The Netherlands

Accepted for publication 18 November 1987

\section{Patients, materials, and methods}

\section{STUDY POPULATION AND ISOLATION OF C TRACHOMATIS}

Specimens for $C$ trachomatis culture were obtained from 185 consecutive patients attending the outpatient clinic for sexually transmitted diseases at the University Hospital Rotterdam-Dijkzigt during the first half of 1986. Specimens for culture were taken from the urethras of 87 adult men (median age 27 , range 18 to 59) who suffered from postgonococcal or nongonococcal urethritis and from the cervices of 98 women (median age 25 , range 16 to 48 ) with complaints of vaginal discharge. Specimens were collected, transported, and cultured as described previously ${ }^{8}$. Briefly, cultures were performed using Hela 229 monolayers that were washed with diethylaminoethyl (DEAE)-dextran in 96 well microtitre plates, incubated for $\mathbf{4 8}$ hours, and stained with fluorescent monoclonal antibodies (Syva). Subpassage was not performed. All available duplicate specimens of the original positive $C$ trachomatis cultures were stored at $-70^{\circ} \mathrm{C}$ and used for passaging and typing in this study.

\section{ANTIGEN PREPARATION AND SEROTYPING BY DOT ELISA}

Duplicates of specimens that yielded a positive result were passaged on McCoy cell monolayers in vials. Serial passage was performed until $100 \%$ infectivity in the monolayer was observed. After a single shell vial 
was sonicated, the culture medium containing suspended $C$ trachomatis and host cell debris was centrifuged at $11000 \times g$ for 15 minutes. The resulting pellet was suspended in phosphate buffered saline (PBS, pH 7.6) containing 0.02\% formalin.

Suspensions of prototype antigens or clinical isolates to be typed were applied to nitrocellulose membranes and dried. The dot ELISA procedure was then performed as described previously. We used 20 different monoclonal antibodies with known serovar specificity. 'Each antigen was scored for colour change on a scale from 0 to $4+$. Serovars were identified by comparing the reaction of each test strain with the pattern of prototype control antigens included in each assay. Criteria for the typing results were applied in accordance with current standard techniques in this laboratory.

\section{Results}

Using the dot ELISA with serovar specific monoclonal antisera, we identified the serovars of $208 \mathrm{C}$ trachomatis strains cultured from 185 patients $(87$ men, 98 women). In three men and 14 women, a second positive culture with the same serovar was also detected at one or two follow up visits after two to 83 (median seven) days. One man and three women had positive cultures with a different serovar at a later visit five to 59 (median 36) days after the first visit. For tabulation purposes, repeat positive cultures were entered only once if the serovar remained the same. A survey of these 190 strains by sex, number, and serovar distribution is given in the table.

In both men and women, the most prevalent serovar was $\mathrm{E}$, followed in men by $\mathrm{D}, \mathrm{F}, \mathrm{H}$, and $\mathrm{K}$, and in women by $\mathrm{F}, \mathrm{K}, \mathrm{D}$, and $\mathrm{H}$. These five serovars were found in $80(89 \%)$ strains from men and $87(87 \%)$ from women. Serovars G, I, I', and J were each present in two to 12 strains. Only one B serovar strain was

Table Distribution of serovars in 190 Chlamydia trachomatis strains of urogenital origin from 185 patients

\begin{tabular}{llll}
\hline \multicolumn{5}{c}{ No of strains from: } \\
\cline { 2 - 4 } Serovar* & $\begin{array}{l}\text { Men } \\
(n=90)\end{array}$ & $\begin{array}{l}\text { Women } \\
(n=100)\end{array}$ & $\begin{array}{l}\text { Total } \\
(n=190)\end{array}$ \\
\hline B & 1 & 0 & 1 \\
D & 19 & 15 & 34 \\
E & 22 & 23 & 45 \\
F & 17 & 22 & 39 \\
G & 0 & 4 & 4 \\
I & 11 & 10 & 21 \\
I & 2 & 2 & 4 \\
I' & 2 & 0 & 2 \\
J & 5 & 7 & 12 \\
K & 11 & 17 & 28 \\
\hline
\end{tabular}

*Serovars $A, B a, C, L_{1}, L_{2}$, and $L_{3}$ were not isolated. detected, and the $A, B a, C, L, L_{2}$, and $L_{3}$ serovars were absent. Two different serovars in one chlamydial culture were detected on two occasions. Serovars E and $F$ were cultured from a male urethral specimen, while serovars $\mathrm{J}$ and $\mathrm{F}$ were isolated from one cervical specimen. Interestingly, a specimen obtained from this patient five days earlier yielded only serovar J.

\section{Discussion}

During previous investigations ${ }^{569}$ serovars $\mathrm{D}, \mathrm{E}$, and $F$ (or combinations of D plus $E$ or $F$ plus $G$ ) were those most commonly found in urogenital infections other than lymphogranuloma venereum (LGV). The sum of the percentages of these serovars was close to $75 \%$ in the United States of America and in some Scandinavian countries. ${ }^{59} \mathrm{~A}$ higher figure $(95 \%)$ reported by Dwyer $e t$ al may have been biased by the small number of strains investigated..$^{10}$ Our results, obtained from Dutch patients, were similar to previous findings in Seattle, with the D plus E serovars present in $42 \%$ and the $F$ serovars in $21 \%$ of strains compared with $47 \%$ and $25 \%$ (including some $\mathrm{G}$ serovar strains) in Seattle. The incidence of the less common serovars $\mathrm{G}(2 \cdot 1 \%)$, $\mathrm{H}(11 \cdot 1 \%)$, I plus $\mathrm{I}^{\prime}(3 \cdot 2 \%)$, and $\mathrm{J}(6 \cdot 3 \%)$ was also within a $5 \%$ range of the percentages found in Seattle, whereas the $\mathrm{K}$ serovar occurred more often in our population $(14.7 \%$ versus $5.9 \%)$. The B serovar, present in 3.9\% in Seattle, ${ }^{9}$ was isolated once in our patients. A few Ba serovars were isolated from genital sources in Seattle ${ }^{11}$ and Denmark, ${ }^{9}$ but were absent from the Dutch population studied. The absence of serovar $\mathrm{C}$ is not surprising as it has seldom been isolated from urogenital specimens. ${ }^{67}$ The serovars were present in roughly equal percentages in men and women in our study. As essentially all the women and about $95 \%$ of the men in this study were heterosexual, cervical strains from the women and urethral strains from the men would be expected to be similar. In contrast, the distribution of serovars in strains found in rectal isolates from homosexual men has been shown to be different from that in cervical isolates. ${ }^{12}$ Thus strains predominating in homosexual men may differ from those found in heterosexuals. We also found no LGV strains in our patients, whereas such strains have been more commonly found in rectal isolates from homosexual men. ${ }^{13}$

As has been reported previously, the dot ELISA method can detect more than one serovar in the same specimen. ${ }^{11}$ In this study simultaneous infection with two serovars was found in two patients. Both patients were highly sexually active and had had multiple partners in the month proceeding testing.

This study shows that a small number of dominantly occurring $(D, E, F, H$, and $K$ ) serovars are primarily responsible for most urogenital infections in 
The Netherlands. These serovars are similar to those that have previously been found to be most common in Seattle and Scandanavia. Our work indicates that the dot ELISA typing system can be applied easily to large scale epidemiological studies and may therefore be of use in assessing temporal and geographical patterns of infection. The fact that most infections are caused by a small number of serovars, however, limits the resolving power of serotyping in more detailed epidemiological studies, such as sexual partner contact tracing. Such studies need a second marker system, with a larger number of distinct types.

We thank Aggie Clark, Linda Cles, Susan Hendricks, and Kim Wong for technical help and Laurie Johnson for typing the manuscript. This study was carried out when Dr Wagenvoort was a visiting faculty member at the University of Washington during leave granted by the University Hospital Rotterdam-Dijkzigt.

\section{References}

1 Centers for Disease Control. Chlamydia trachomatis infections: policy guidelines for prevention and control. MMWR 1985;34 suppl:53-74.

2 Stamm WE, Holmes KK. Chlamydia trachomatis infections of the adult. In: Holmes KK, Mårdh P-A, Sparling PF, Wiesner PJ, eds. Sexually transmitted diseases. New York: McGraw-Hill, 1984:258-70.

3 Thompson S, Washington E. Epidemiology of sexually transmit- ted Chlamydia trachomatis. Epidemiol Rev 1983;5:96-123.

4 Washington AE, Johnson RE, Sanders LL. Chlamydia trachomatis infections in the United States. What are they costing us? JAMA 1987;257:2070-2.

5 Wang SP, Grayston JT, Kuo CC, Alexander ER, Holmes KK. Serodiagnosis of Chlamydia trachomatis infection with the microimmunofluorescence test. In: Hobson D, Holmes $\mathrm{K}$, eds. Nongonococcal urethritis and related infections. Washington DC: American Society for Microbiology, 1977:237-48.

6 Wang SP, Kuo CC, Barnes RC, Stephens RS, Grayston JT. Immunotyping of Chlamydia trachomatis with monoclonal antibodies. J Infect Dis 1985;152:791-800.

7 Barnes RC, Wang SP, Kuo CC, Stamm WE. Rapid immunotyping of Chlamydia trachomatis with monoclonal antibodies in a solid-phase enzyme-immunoassay. J Clin Microbiol 1985; 22:609-13.

8 Tjiam KH, van Heijst BYM, van Zuuren A, et al. Evaluation of an enzyme immunoassay for the diagnosis of chlamydial infections in urogenital specimens. J Clin Microbiol 1986;23:752-4.

9 Kuo CC, Wang SP, Holmes KK, Grayston JT. Immunotypes of Chlamydia trachomatis isolates in Seattle, Washington. Infect Immun 1983;41:865-8.

10 Dwyer RStC, Treharne JC, Jones BR, Herring J. Chlamydial infection: results of microimmunofluorescence tests for the detection of type-specific antibody in certain chlamydial infections. British Journal of Venereal Diseases 1972;48:452-9.

11 Barnes RC, Suchland RJ, Wang SP, Kuo CC, Stamm WE. Detection of multiple serovars of Chlamydia trachomatis in genital infections. J Infect Dis 1985;152:905-9.

12 Barnes RC, Rompalo A, Stamm WE. Comparison of Chlamydia trachomatis serovars causing rectal and cervical infection. $J$ Infect Dis 1987;156:953-8.

13 Quinn TC, Goodell SE, Mkrtichian E, et al. Chlamydia trachomatis proctitis. $N$ Engl J Med 1981;305:195-200. 